\title{
Final Report: Investigation of Saturated Degenerate Four-Wave Mixing Spectroscopy For Quantitative Concentration Measurements
}

\author{
Grant No. DE-FG02-94ER14469, August, 1997 - January, 1999
}

Principal Investigator: Robert P. Lucht (rlucht@tamu.edu)

Dept. of Mechanical Engineering, University of Ilinois, Urbana, IL 61801

Graduate Students Supported:

Thomas A. Reichardt and William C. Giancola

\section{PROGRAM SCOPE}

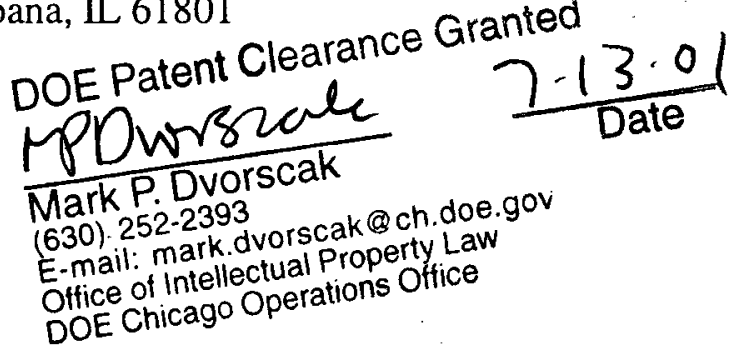

Degenerate four-wave mixing (DFWM) and polarization spectroscopy (PS) are techniques that shows great promise for sensitive measurements of transient gas-phase species, and diagnostic applications of DFWM are being pursued actively at laboratories throughout the world. However, significant questions remain regarding strategies for quantitative concentration measurements using these techniques. The primary objective of this research program is to develop and test strategies for quantitative concentration measurements in flames and plasmas. Theoretically, we are investigating the physics of the PS and DFWM processes by direct numerical integration (DNI) of the time-dependent density matrix equations that describe the four-wave mixing interaction. Significantly fewer restrictive assumptions are required when the density matrix equations are solved using this DNI approach compared with the assumptions required to obtain analytical solutions. For example, the Zeeman structure of the resonance and effects such as Doppler broadening can be included, both the pump and probe beams can be saturating in the DNI calculations. As computer processing speeds have increased, we have incorporated more complicated physical models into our DNI codes.

The incorporation of the Zeeman state structure of the laser-coupled energy levels has played a very significant role in our theoretical efforts over the last three years. For example, it has enabled us to develop theoretical models of the PS process. During the last three years of the grant we (1) developed a computer code for single-mode PS and investigated the effects of collisions and Doppler broadening ; (2) investigated the effects of multi-axial-mode laser radiation on PS signal generation, (3) performed an extensive investigation of saturation effects in DFWM for different polarization configurations, (4) began a numerical study of picosecond PS. The incorporation of the Zeeman state structure in our DNI codes has given us a very powerful tool for the investigation of molecular spectroscopy, and much of our proposed future work is based upon this capability. Our diagnostic experiments are performed in wellcharacterized flames to assess the potential of the techniques for quantitative concentration measurements, and for meaningful comparison with our theoretical results. 


\section{DISCLAIMER}

This report was prepared as an account of work sponsored by an agency of the United States Government. Neither the United States Government nor any agency thereof, nor any of their employees, makes any wartanty, express or implied, or assumes any legal liability or responsibility for the accuracy, completeness, or usefulness of any information, apparatus, product, or process disclosed, or represents that its use would not infringe privately owned rights. Reference herein to any specific commercial product, process, or service by trade name, trademark, manufacturer, or otherwise does not necessarily constitute or imply its endorsement, recommendation, or favoring by the United States Government or any agency thereof. The views and opinions of authors expressed herein do not necessarily state or reflect those of the United States Government or any agency thereof. 


\section{DISCLAIMER}

Portions of this document may be illegible in electronic image products. Images are produced from the best available original document. 


\section{THEORETICAL AND EXPERIMENTAL INVESTIGATION OF POLARIZATION SPECTROSCOPY}

\section{Theoretical Analysis of Single-Mode Polarization Spectroscopy}

The physics of polarization spectroscopy (PS) was investigated by direct numerical integration of the time-dependent density matrix equations (Reichardt and Lucht, 1998). The Zeeman structure of the upper and lower energy levels was included in a multi-state formulation of the density matrix equations. The numerical solution of the time-dependent density matrix equations enabled us to investigate the effects of strong saturation on PS signal levels and line. shapes. Bath levels not directly coupled by the laser radiation were included in the numerical modeling to investigate the effects of collisional rates and different types of collisions on signal levels and line shapes. The effects of Doppler broadening were included by solving the density matrix equations for numerous velocity groups.

At low laser power we found that the homogeneously broadened PS lineshape for the case where the pump and probe have the same frequency is Lorentzian-cubed as compared to the Lorentzian predicted in several previous low power analytical solutions. As the pump laser intensity increases, the PS signal initially is proportional to the square of the pump intensity (for constant probe intensity) and reaches a peak level when the pump beam intensity is approximately equal to the saturation intensity. In this low laser power regime, the line-center PS signal is proportional to (collision rate) ${ }^{-6}$, obviously greatly complicating the application of unsaturated PS for quantitative concentration measurements in flames. As the transition begins to saturate at higher laser intensities, the dependence of the PS signal level on the collision rate decreases drastically, although the line-center PS signal is still proportional to (collision rate)-2. For transitions where the Doppler width is larger than the collisional width, however, the PS signal is nearly independent of the collision rate when the pump laser beam intensity is approximately equal to the saturation intensity for the transition, as shown in Fig. 1.

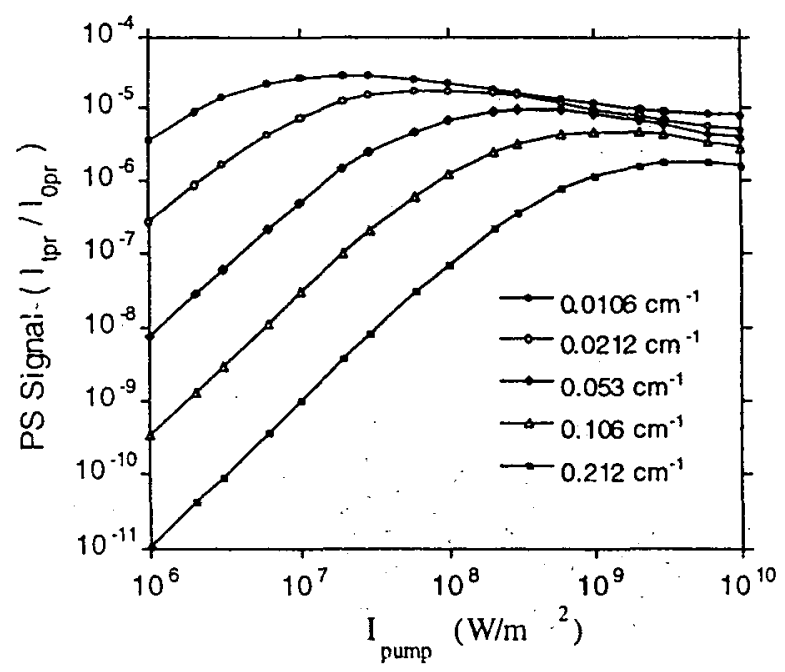

Fig. 1. Saturation curves for the line-center PS signal from the R(4) resonance for five different values of the homogeneous (collisional) linewidth and for a Doppler width of $0.1 \mathrm{~cm}^{-1}$. 


\section{Polarization Spectroscopy Measurements of $\mathrm{OH}$ in Well-Characterized Flames}

Polarization spectroscopy (PS) line shapes and signal intensities were measured experimentally in well-characterized hydrogen/air flames operated a wide range of equivalence ratios (Reichardt et al., 2000a). The PS apparatus is shown schematically in Fig. 1. Both low (perturbative) and high (saturating) pump beam intensities in the counterpropagating probe geometry. The results of the saturated PS measurements of $\mathrm{OH}$ concentration in a series of nearadiabatic flames at equivalence ratios ranging from 0.5 to 1.5 are shown in Fig. 2. For the results shown in Fig. 1 the $P_{1}(2)$ transition in the $A^{2} \Sigma^{+}-X^{2} \Pi$ electronic transition was probed. The PS measurements were calibrated at a single equivalence ratio $(\phi=0.6)$ using the adiabatic equilibrium $\mathrm{OH}$ concentration. The use of saturating pump intensities minimizes the effect of pump beam absorption, providing more accurate number density measurements. The saturated PS number density measurements are in excellent agreement with adiabatic equilibrium calculations of $\mathrm{OH}$ number density for lean flames, although the PS results are 30-50\% higher for the richest equivalence ratio. The PS measurements are also in excellent agreement with laser àbsorption measurements for lean flame conditions (a diffusion flame surrounds the inner premixed flame for rich flame conditions and is responsible for the disagreement between the laser absorption measurements and adiabatic equilibrium calculations).

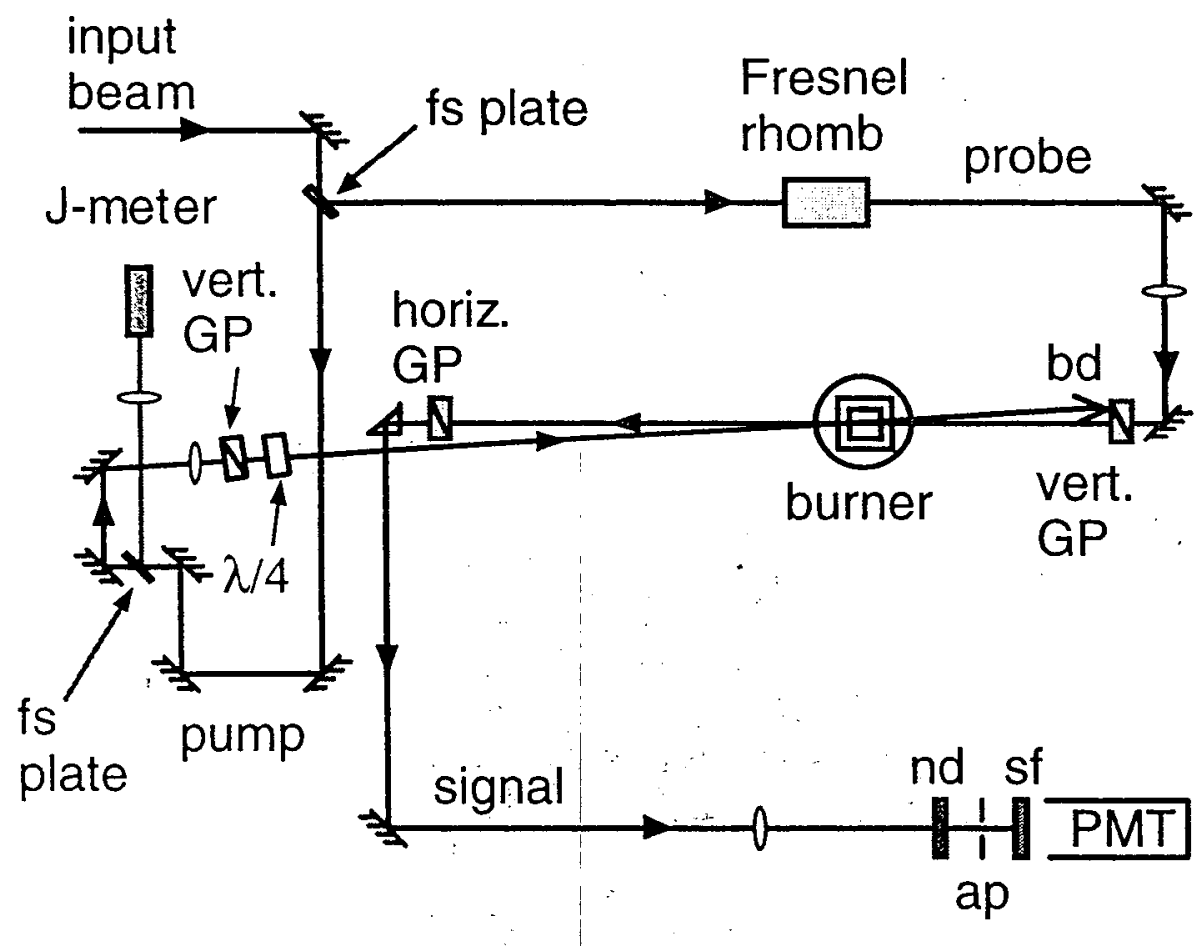

Fig. 2. Schematic diagram of the PS experiment. J-meter - Joulemeter, fs plate - fused-silica plate, GP - Glan polarizer, bd - beam dump, nd - neutral density filter, ap - aperture, sf - spectral filter, $\lambda / 4$ - quarterwave plate, PMT - photomultiplier tube. 


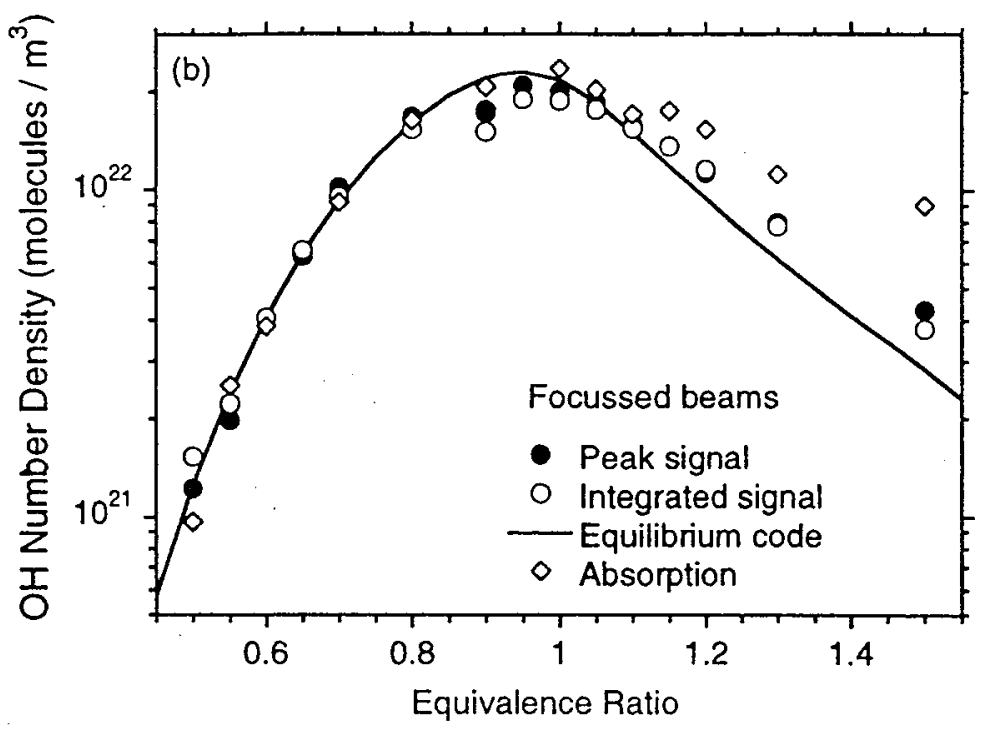

Fig. 3. OH concentrations in near-adiabatic hydrogen/air flames measured using saturated PS and laser absorption. The experimental measurements are compared with $\mathrm{OH}$ concentrations at adiabatic equilibrium.

The effects of saturation on the line-center signal intensity and the resonance line width were also investigated. Low-power and high-power (saturated) PS line shapes are compared in Fig. 4 with the absorption line shape for the $\mathrm{P}_{1}(2)$ resonance. As the resonance saturates, the PS linewidth increases until it is nearly equal to the absorption linewidth, and remains nearly unchanged up to the highest intensities that we investigated. For laser intensities significantly in excess of the saturation intensity, the DFWM linewidth is much greater than the PS linewidth. The fact that the linewidth does not continue to broaden as the laser power is potentially a great advantage for PS as compared to DFWM or to LIF, permitting excitation of isolated transitions even at very high laser intensities. This may be particularly important for infrared PS because of the spectral congestion of this region.

\section{Multi-Axial-Mode Effects in Polarization Spectroscopy}

The effects of multi-frequency-mode laser radiation on polarization spectroscopy signal generation were investigated by direct numerical integration of the time-dependent density matrix equations (Giancola et al., 2000). The numerical solution of the density matrix equations allows us to incorporate a physically reasonable model for a pulsed dye laser radiation in our analysis of the laser-resonance interaction. The laser radiation is modeled as the sum of electric fields from a finite number of modes that are assumed to have random pulse-to-pulse phases. The amplitude of each mode on a given laser pulse is randomly distributed about a mean mode amplitude. The mean amplitude is dependent on the frequency detuning of the mode from the central laser frequency. Calculations were performed for a homogeneously broadened resonance (collisional 


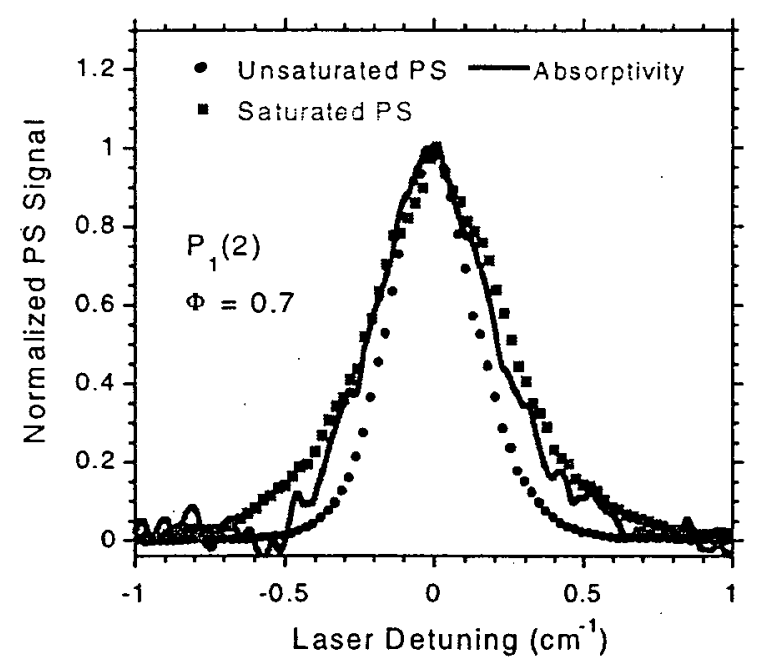

Fig. 4. PS line shapes for saturating and non-saturating pump intensities compared with the absorption line shape for the $P_{1}(2)$ resonance.

broadening only) and for a resonance that was both collision- and Doppler-broadened. The effect of the multi-mode laser radiation on polarization spectroscopy line shapes and saturation curves was investigated parametrically for different values of the laser bandwidth and mode spacing and resonance collision and Doppler widths. The saturation parameter for the resonance is strongly dependent on the ratio of the laser bandwidth to resonance collision width when the laser bandwidth is greater than the collision width. The pulse-to-pulse fluctuation in polarization spectroscopy signal levels is found to decrease substantially for saturation pump intensities. The inclusion of the multi-mode laser structure into our density matrix equations represents a significant advance in modeling the nonlinear interaction of laser radiation with atomic or molecular resonances.

The effect of the ratio of the laser linewidth and the homogeneous (collisional) linewidth on the saturation behavior and noise statistics of a homogeneously broadened PS resonance is shown in Fig. 5. The points shown are the results of single-pulse calculations for the multi-axialmode laser, and the lines are the results of single-mode calculations. The multi-mode calculations were performed for a laser linewidth of $0.1 \mathrm{~cm}^{-1}$ with a mode spacing of $0.025 \mathrm{~cm}^{-1}$. When the laser linewidth is greater than the collisional linewidth, the effective saturation intensity for the resonance increases and the pulse-to-pulse fluctuations in PS signal intensity increase dramatically compared to the case where the laser and collisional widths are comparable. Note the huge pulse-to-pulse PS signal fluctuations for the case where $\Delta \omega_{c}=0.01$ $\mathrm{cm}^{-1}$, ten times less than the laser linewidth, and that the single-pulse signal levels are always less for the multi-mode case than for the single-mode case. It is also very interesting to note that when the collisional linewidth is significantly greater than the multi-mode laser linewidth, the single-pulse multi-mode PS signal levels are always greater than the single-mode PS signal levels. When the collisional linewidth is greater than the laser linewidth the resonant transition can respond to even the fastest fluctuations in the laser intensity, and the PS signal is enhanced by the multi-mode intensity spiking. 


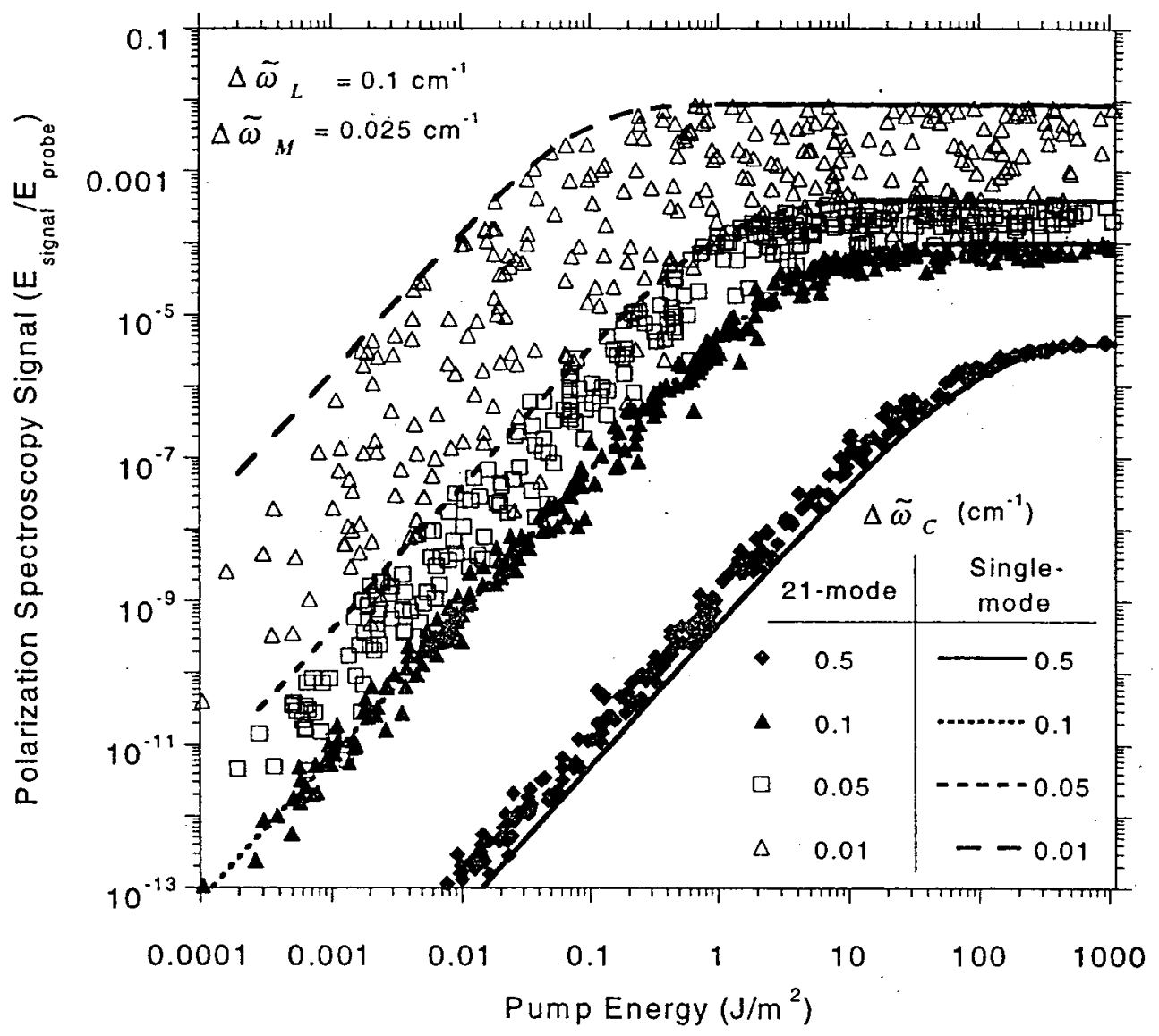

Fig. 5. PS saturation curves for the $P_{I}(2)$ resonance for a laser linewidth of $0.1 \mathrm{~cm}-1$ and different values of the collisional linewidth.

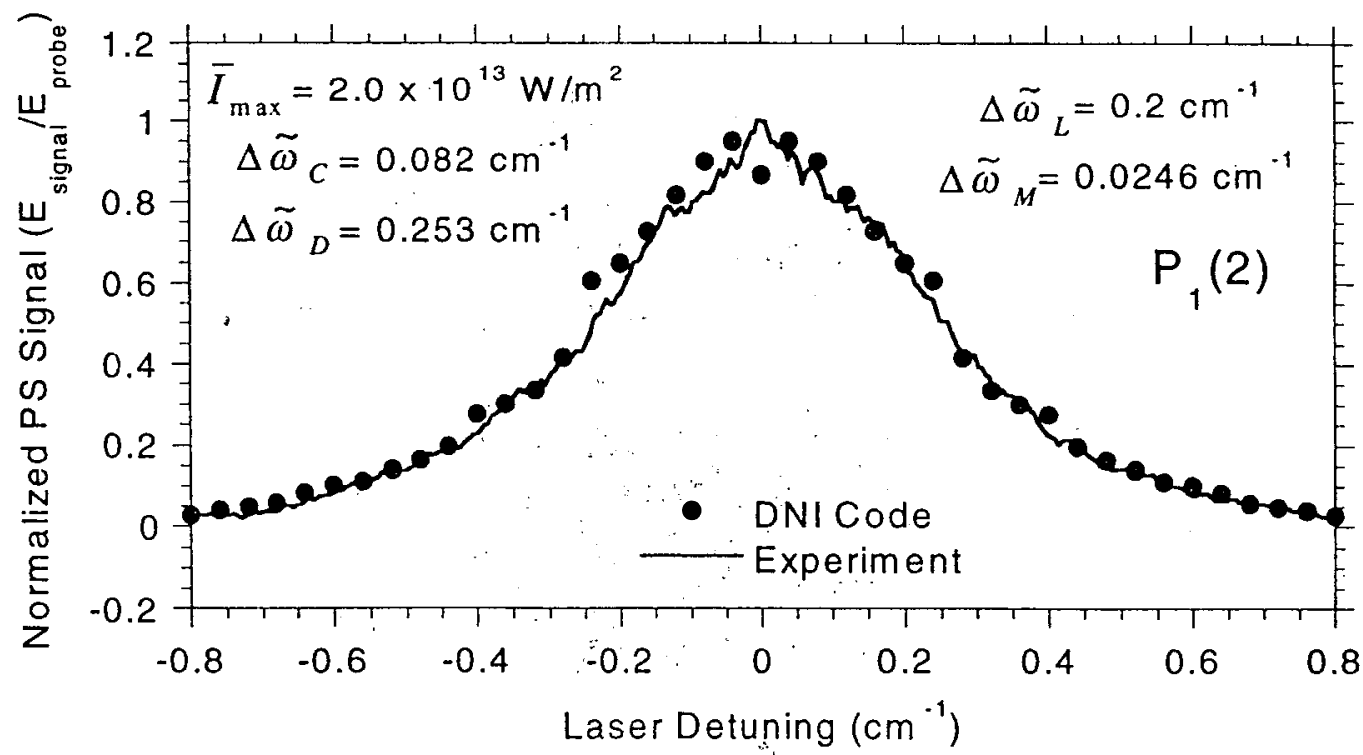

Fig. 6. Comparison of calculated and measured PS line shape for a multi-axial-mode laser at high saturation. 


\section{$\underline{\text { Picosecond Polarization Spectroscopy }}$}

Our initial work in this area is discussed in detail in the proposal and in an article submitted to the Journal of Chemical Physics (Reichardt et al., 2000b).

\section{THEORETICAL AND EXPERIMENTAL INVESTIGATION OF DFWM}

\section{DFWM Measurements in Well-Characterized Flames}

Degenerate four-wave mixing (DFWM) line shapes and signal intensities were measured experimentally in well-characterized hydrogen/air flames operated over a wide range of equivalence ratios (Reichardt et al., 1999). Both low (perturbative) and high (saturating) beam intensities in the phase-conjugate geometry were investigated. Resonances in the $A^{2} \Sigma^{+}-X^{2} \Pi$ $(0,0)$ band of $\mathrm{OH}$ were probed using multi-axial-mode laser radiation. The effects of saturation on the line-center signal intensity and the resonance linewidth were investigated. The shape of the saturation curve was compared with a number of simplified analytical models.

The DFWM signal intensities were used to measure $\mathrm{OH}$ number densities in a series of near-adiabatic flames at equivalence ratios ranging from 0.5 to 1.5 . The use of saturating pump intensities was shown to minimize the effects of beam absorption, providing more accurate number density measurements. The results of the saturated DFWM measurements are shown in Fig. 7. The saturated DFWM results are in excellent agreement with $\mathrm{OH}$ absorption measurements in lean flames and adiabatic equilibrium calculations of $\mathrm{OH}$ number density at all equivalence ratios. The saturated DFWM measurements were performed at laser intensities where the DFWM signal should be fairly insensitive to changes in collisional and Doppler widths

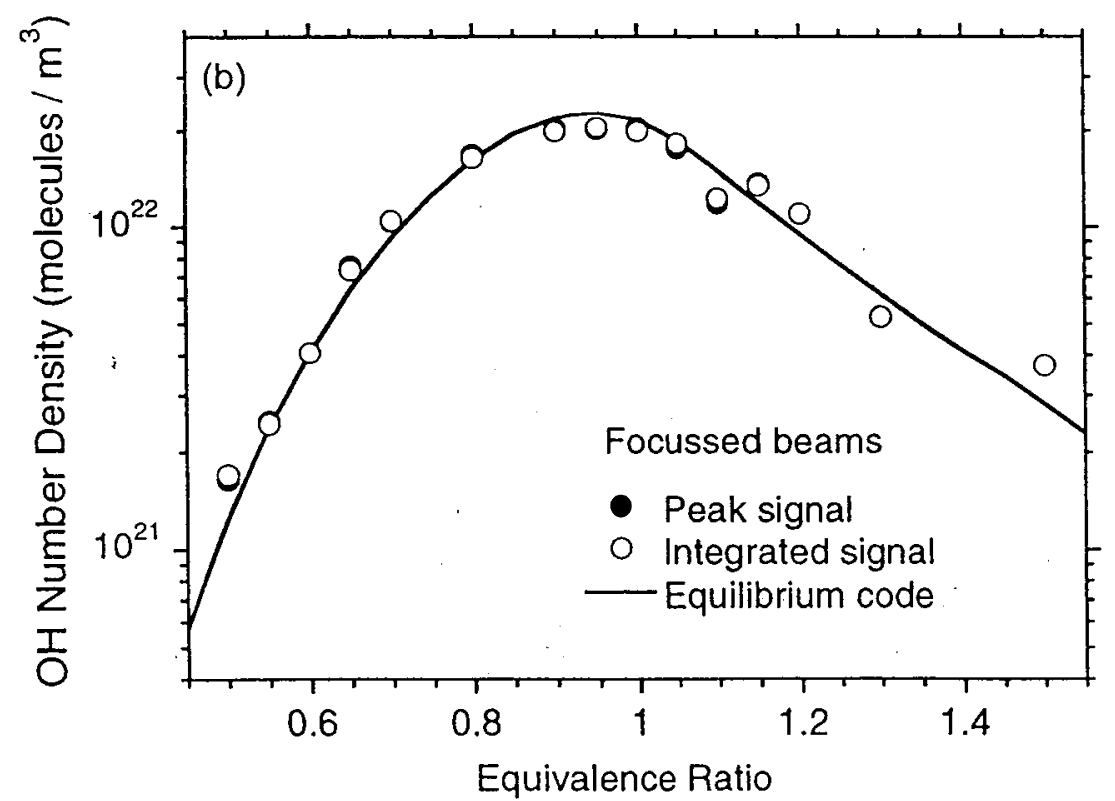

Fig. 7. OH concentrations in near-adiabatic hydrogen/air flames measured using saturated DFWM. The experimental measurements are compared with predicted $\mathrm{OH}$ concentrations at adiabatic equilibrium. 
according to the simplified analytical model of Reichardt and Lucht (1996). In comparison to the saturated PS measurements, the saturated DFWM were in excellent agreement with $\mathrm{OH}$ concentrations calculated from adiabatic equilibrium at all equivalence ratios, whereas the saturated PS measurements were systematically higher than adiabatic equilibrium concentrations at the richest equivalence ratios. The saturated DFWM measurements were far more accurate than low-intensity, unsaturated DFWM measurements because the unsaturated measurements were affected significantly by absorption of the pump and probe beams.

The polarization dependence of the $P_{1}(2)$ and $R_{2}(1)$ resonances is investigated in both laser intensity regimes and the relative signal intensities agree with results obtained by direct numerical integration of the density matrix equations. As shown in Fig. 8, there is a significant change in relative reflectivities for different polarization configurations when saturated.
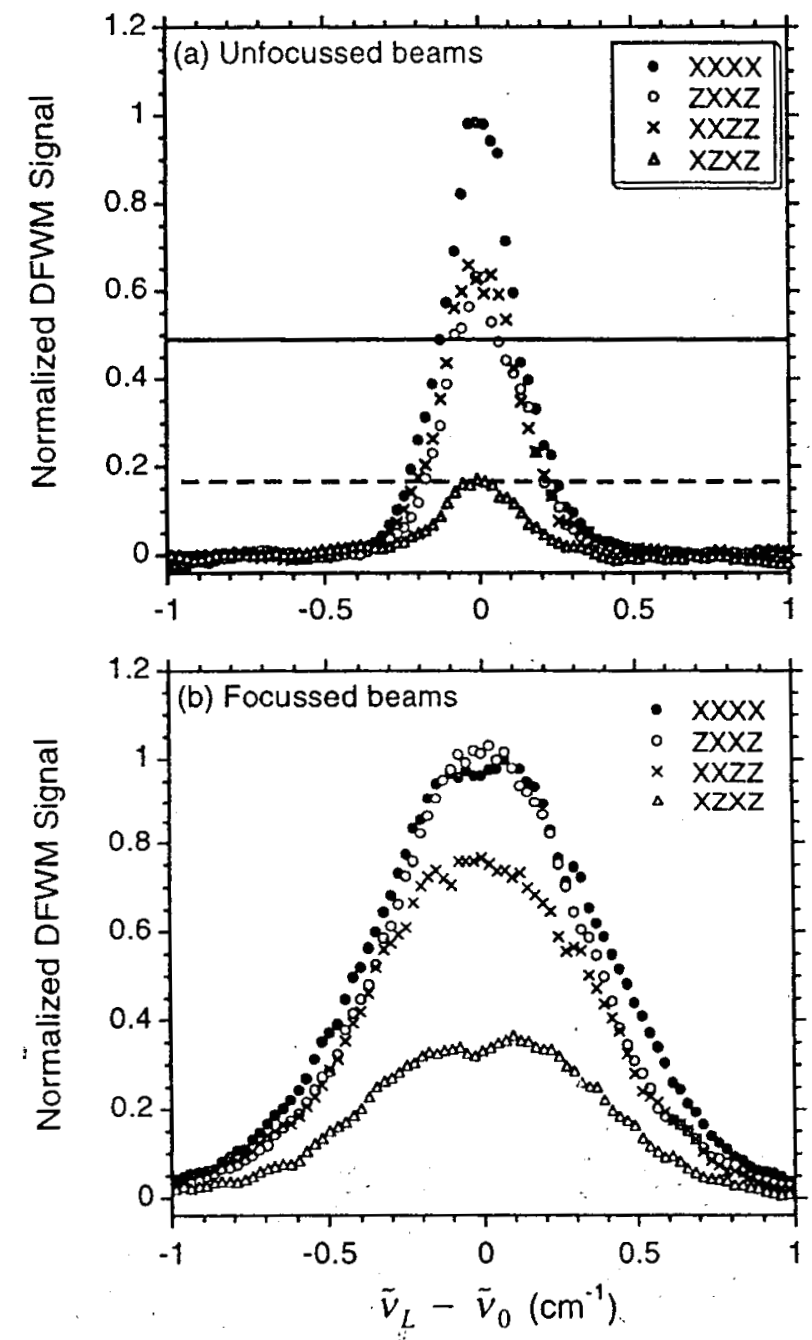

Fig. 8. DFWM lineshapes of the $P_{1}(2)$ resonance for (a) unfocussed and (b) focussed beams for different polarization geometries. These lineshapes were acquired in the $\phi=0.7$ flame. The solid line indicates the theoretical signal level in the perturbative limit for the $\mathrm{ZXXZ}$ and $\mathrm{XXZZ}$ configurations and the dashed line indicates the theoretical signal level for the XZXZ configuration. 


\section{DNI Calculation of Polarization and Saturation Effects in DFWM Spectroscopy}

The physics of the degenerate four-wave mixing process for resonant transitions between two degenerate energy levels was investigated by direct numerical integration of the timedependent density matrix equations (Reichardt and Lucht, 1999). The Zeeman structure of the upper and lower energy levels was included in a multi-state formulation of the density matrix equations. The inclusion of the Zeeman structure enabled the investigation of the DFWM process for different polarization configurations of the forward pump, backward pump, and probe beams. This study represents a major step forwards in terms of realistic modeling of DFWM as a diagnostic probe; so-called crossed polarization configurations are used commonly to reduce scattered light and to discriminate against thermal gratings. The theory of Williams et al. (1994) applies in the perturbative regime but not in the saturation regime.

In our study, saturation curves and line shapes were calculated for different polarization configurations and for numerous low-J transitions. At low laser intensity, the results of our calculations are in excellent agreement with perturbation theory in terms of the relative intensities of the DFWM signal for linear polarization configurations. As the laser intensity increases and the resonance starts to saturate, we found in general that the relative DFWM reflectivity increases for the crossed polarization (CP) configurations compared to the parallel polarization (PP) configuration because the saturation intensity is higher. However, for some resonance transitions, some of the $\mathrm{CP}$ configurations saturate at lower laser intensities than the PP configuration, even though the reflectivity for these $\mathrm{CP}$ configurations is much lower than for the PP configuration in the perturbative intensity limit. This is shown for the $R(1)$ transition in Fig. 9. The ZXZX/ZZZZ ratio of reflectivities decreases as the laser intensity increase because as shown in Fig. 10 the Zeeman transitions $\left(M_{g}= \pm 1 \leftrightarrow M_{e}= \pm 1\right)$ saturate and deplete the lower level population without contributing to the DFWM signal (notation such as ZXZX indicates direction of the linear polarizations of the forward pump, probe, backward pump and signal, respectively). The effect of saturation on the resonance line shapes for the different polarization configurations was also investigated.

A limited number of calculations are performed for resonances that are Doppler broadened as well as collision broadened. The effect of saturation on the reflectivity of the $\mathrm{CP}$ configurations compared to the PP configuration was even more significant for resonances with comparable Doppler and collisional broadening. This is shown in Fig. 11 for the R(1) transition for a Doppler width of $0.1 \mathrm{~cm}-1$ and a collisional width of $0.053 \mathrm{~cm}-1$. In this case the DFWM signal for the ZXXZ configuration actually becomes greater than the ZZZZ signal as the resonance saturates. This behavior is also evident in the experimental data shown in Fig. 8, and indicates that in many cases there is little or no signal penalty associated with the use of $\mathrm{CP}$ polarization configurations. 


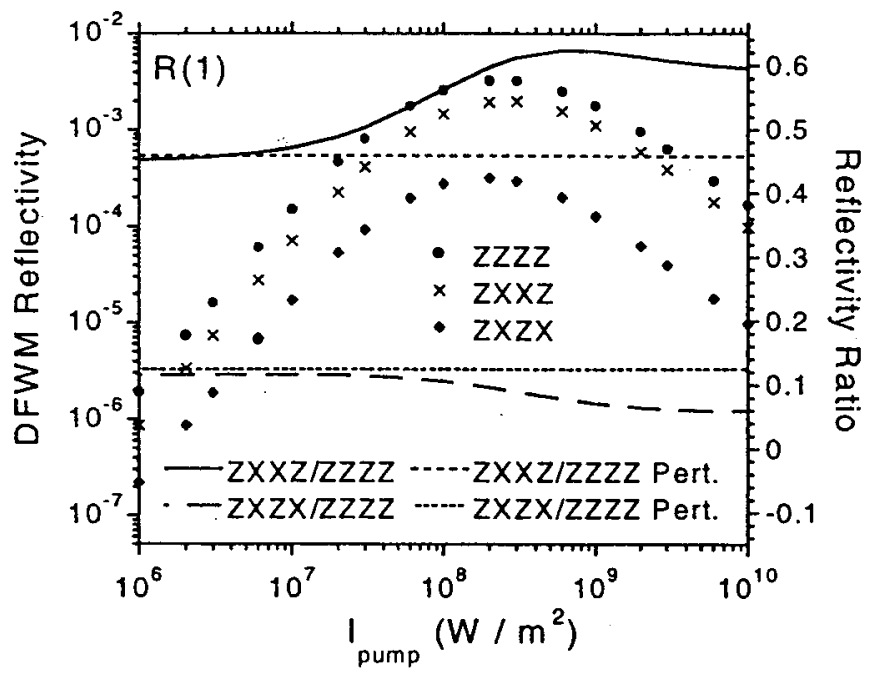

Figure 9. Saturation curves for the R(1) transition for the ZZZZ, ZXZX, and ZXXZ polarization configurations. The probe and pump intensities are equal. The backward and forward pump beams intensities are equal.

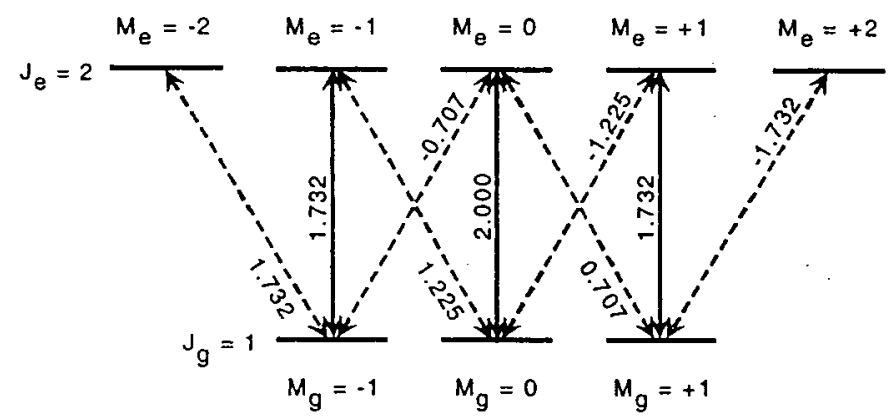

Figure 10. Energy level schematic of the $\mathrm{R}(1)$ transition. The allowed $\Delta M=0$ transitions are coupled by $z$ polarized light and are indicated with solid arrows. The allowed $\Delta M= \pm 1$ transitions are coupled by $\mathrm{x}$-polarized light and are indicated with dashed arrows.

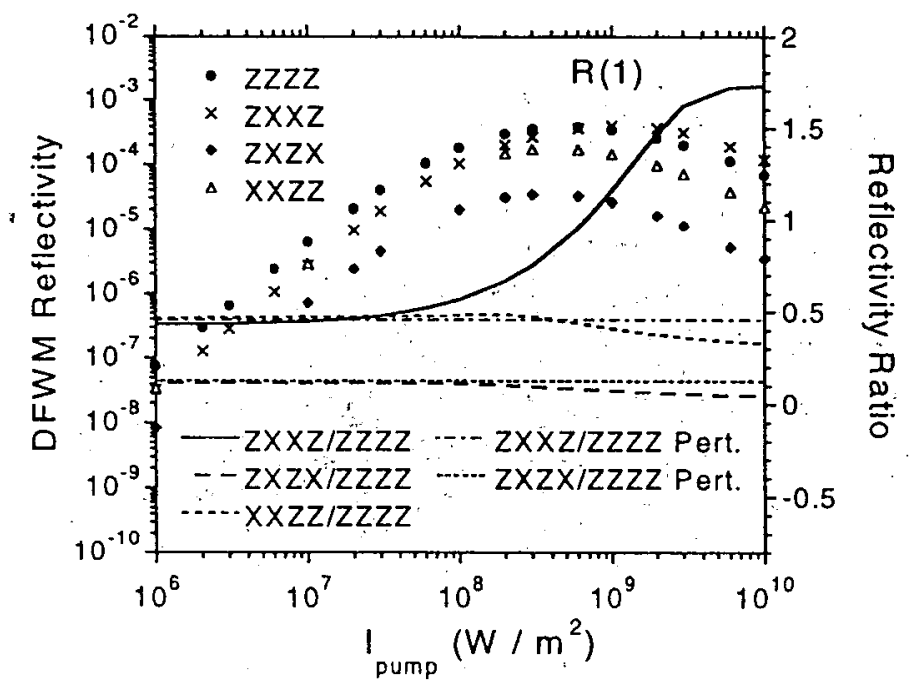

Figure 11. Saturation curves for the R(1) transition for the ZZZZ, ZXXZ, ZXZX, and XXZZ polarization configurations. The Doppler width of resonance is set equal to $0.1 \mathrm{~cm}^{-1}$. 


\section{REFERENCES}

W. C. Giancola, T. A. Reichardt, and R. P. Lucht (2000), J. Opt. Soc. America B, submitted for publication.

T. A. Reichardt and R. P. Lucht (1996), J. Opt. Soc. Am. B 13, 1107-1119.

T. A. Reichardt and R. P. Lucht (1998), J. Chem. Phys. 109, 5830-5843.

T. A. Reichardt and R. P. Lucht (1999), J. Chem. Phys. 111, 10008-10020.

T. A. Reichardt, W. C. Giancola, C. M. Shappert, and R. P. Lucht (1999), Appl. Opt. 38, 69516961.

T. A. Reichardt, W. C. Giancola, and R. P. Lucht (2000a), Appl. Opt., accepted for publication, to appear in April 20 issue.

T. A. Reichardt, F. Di Teodoro R. L. Farrow, S. Roy, and R. P. Lucht (2000b), J. Chem. Phys., submitted for publication.

S. Williams, R. N. Zare, and L.A. Rahn (1994), J. Chem. Phys. 101, 1072-1092.

\section{DOE/BES PROJECT PUBLICATIONS 1997-2000}

1. T. A. Reichardt and R. P. Lucht, "Interaction of Closely Spaced Resonances in Degenerate Four-Wave Mixing Spectroscopy," Journal of the Optical Society of America B 14, 24492458 (1997).

2. T. A. Reichardt and R. P. Lucht, "Theoretical Calculation of Lineshapes and Saturation Effects in Polarization Spectroscopy," Journal of Chemical Physics 109, 5830-5843 (1998).

3. T. A. Reichardt and R. P. Lucht, "Investigation of the Forward Phase-Matched Geometry for Degenerate Four-Wave Mixing Spectroscopy," Journal of the Optical Society of America B 15, 2566-2572 (1998).

4. T. A. Reichardt and R. P. Lucht, "Resonant Degenerate Four-Wave Mixing Spectroscopy of Transitions with Degenerate Energy Levels: Saturation and Polarization Effects," Journal of Chemical Physics 111, 10008-10020 (1999).

5. T. A. Reichardt, W. C. Giancola, C. M. Shappert, and R. P. Lucht, "Experimental Investigation of Saturated Degenerate Four-Wave Mixing for Quantitative Concentration Measurements," Applied Optics 38, 6951-6961 (1999).

6. T. A. Reichardt, W. C. Giancola, and R. P. Lucht, "Experimental Investigation of Saturated Polarization Spectroscopy for Quantitative Concentration Measurements," Applied Optics, accepted for publication, to appear in April 20 issue (2000).

7. W. C. Giancola, T. A. Reichardt, and R. P. Lucht, "Multi-Axial-Mode Laser Effects in Polarization Spectroscopy," Journal of the Optical Society of America B, submitted for publication (2000).

8. T. A. Reichardt, F. Di Teodoro R. L. Farrow, S. Roy, and R. P. Lucht, "Collisional Dependence of Polarization Spectroscopy with a Picosecond Laser,". Journal of Chemical Physics, submitted for publication (2000). 
VII. GRADUATE DISSERTATIONS RESULTING FROM DOE/BES SUPPORT 1997 2000

1. Thomas A. Reichardt, "Investigation of Degenerate Four-Wave Mixing and Polarization Spectroscopy for Quantitative Measurements in Combustion Environments," Ph.D. Thesis (1999).

2. William C. Giancola, "Theoretical Investigation of Polarization Spectroscopy in MultiAxial-Mode Fields," M.S. Thesis (1999). 